\title{
Qualidade dos bulbos de cebola 'Superex' armazenados sob refrigera- ção, quando expostos à condição ambiente
}

\author{
Ana Carolina Almeida Miguel; José Fernando Durigan \\ UNESP-FCAV, Depto. Tecnologia, Via de acesso Prof. Paulo Donato Castellane, s/n, 14884-900 Jaboticabal-SP; \\ anaamiguel@yahoo.com.br; jfduri@fcav.unesp.br
}

\section{RESUMO}

O armazenamento refrigerado da cebola tem sido recomendado como ideal, mas os produtores sempre questionam sobre a qualidade comercial e o consumo dos bulbos durante este período e depois de levados à condição ambiente. Avaliou-se a qualidade de bulbos de cebola 'Superex' acondicionados em sacos de rede de fibra plástica e armazenados a $10-12^{\circ} \mathrm{C}(68 \%$ UR) por até 91 dias, quando levados à condição ambiente. Após $1 ; 16 ; 31 ; 61$ e 91 dias foram tomadas amostras, ao acaso, que eram levadas à condição ambiente $\left(22^{\circ} \mathrm{C}, 59 \% \mathrm{UR}\right)$. Estas cebolas foram avaliadas quanto à massa fresca, aparência e ocorrência de podridões, e quanto à coloração interna, luminosidade, ângulo de cor e cromaticidade, atividade da aliinase e teores de sólidos solúveis e de acidez titulável. Os bulbos, quando levados ao ambiente, perderam massa com maior intensidade do que quando armazenados sob refrigeração e mantiveram qualidade aceitável por até 38 dias. Somente os armazenados sob refrigeração por 91 dias tinham aparência ruim. Os bulbos analisados apresentaram tendência ao amarelecimento interno, sem alteração significativa nos teores de SS e de AT. Durante o período refrigerado a pungência dos mesmos aumentou, de "pungente" para "picante", que se manteve estável durante o armazenamento ao ambiente.

Palavras-chave: Allium cepa, armazenamento refrigerado, pungência, aliinase.

\begin{abstract}
Quality of onion bulbs cv. Superex stored under refrigeration, when exposed to environmental conditions

Cold storage has been recommended to preserve onion bulbs, but producers always have doubts about its quality, during this period and when they are exposed to environmental conditions. We evaluated the onion bulbs quality, cv. Superex, packed in plastic fiber bags, during storage under $10-12^{\circ} \mathrm{C}(68 \% \mathrm{RH})$ during 91 days. After $1 ; 16 ; 31 ; 61$ and 91 days, samples were transferred to environmental conditions $\left(22^{\circ} \mathrm{C}, 59 \% \mathrm{RH}\right)$. Onion bulbs were evaluated for fresh mass, appearance and occurrence of rot, internal color, lightness, color or hue angle and chromaticity, alliinase activity, soluble solids and titratable acidity content. Bulbs under environmental condition lost more fresh mass than those stored under refrigeration and maintained acceptable quality for 38 days. Only bulbs stored during 91 days presented bad appearance when transferred to environmental conditions. Bulbs showed tendency of internal yellowing, without significant changes in soluble solids and titratable acidity. During the cold storage period, the pungency increased from "pungent" to "hot pungent" and remained stable during ambiental conditions.
\end{abstract}

Keywords: Allium cepa, refrigerated storage, pungency, alliinase.

(Recebido para publicação em 8 de maio de 2006; aceito em 19 de maio de 2007)

\begin{abstract}
$\mathrm{A}$ cebola ocupa no Brasil o terceiro lugar dentre as hortaliças de maior importância econômica. Dentre as aliáceas, tem a maior área cultivada e está presente em todas as regiões brasileiras, excetuando-se a Norte. Juntamente com o alho, é a cultura condimentar de maior importância e consumo (Oliveira, 2004). O consumo da cebola no Brasil apresentou tendência de aumento, devido principalmente aos produtos processados. Em virtude do aumento da população urbana e da maior participação da mulher no mercado de trabalho, verificou-se uma redução no consumo desta olerácea no âmbito dos domicílios e, em contrapartida, um incremento do setor institucional. Por outro lado, é elevada a elasticidade-preço da oferta da cebola, característica relevante nos processos de produção e comercialização que exprimem a sensibilidade da pro-
\end{abstract}

dução às variações de preços (Vilela et al., 2005).

Cebolas conservadas em depósitos, em condição ambiente, apodrecem facilmente, dependendo do grau de higienização do recinto e da cultivar (Varela et al., 1980). Seu armazenamento, por períodos relativamente longos, exige o uso de ambientes devidamente controlados, para evitar degradações indesejáveis e assegurar o abastecimento contínuo do mercado. Sendo a cebola basicamente utilizada como agente aromatizante de outros alimentos, a evolução do seu odor característico ou pungência durante o período de estocagem deve ser conhecida (Andrade \& Lima, 1983), permitindo sua melhor utilização, tanto in natura como processada (Kopsell \& Randle, 1997). De acordo com Rubatzky \& Yamaguchi (1997), a qualidade dos bul- bos é avaliada pela pungência e conteúdos de massa fresca e de sólidos solúveis. Tais características variam com a cultivar, podendo ou não ser afetadas pela época de colheita. A pungência característica e sua intensidade relacionam-se com o conteúdo de compostos sulfurados e seus derivados, ácido sulfênico, amônia e ácido pirúvico (Randle \& Bussard, 1993; Randle et al., 1994).

Miguel et al. (2004) sugerem que as cebolas podem ser classificadas mediante a combinação entre os teores de sólidos solúveis (SS) e de acidez titulável (AT) e da atividade da aliinase, indicando que uma cebola, para ter sabor considerado "picante" deve ter alta atividade da aliinase $(>6,0$ mmoles ác. Pirúvico $\left.\mathrm{g}^{-1}\right)$, elevada acidez $(>0,3 \mathrm{~g}$ ác. pirúvico $100 \mathrm{~g}^{-1}$ ) e baixa concentração de sólidos $\left(<6^{\circ}\right.$ Brix $)$; para sabor "modera- 
damente picante" deve ter valores intermediários de sólidos solúveis (6$8^{\circ}$ Brix), acidez (0,3-0,2 g ác. pirúvico $\left.100 \mathrm{~g}^{-1}\right)$ e atividade da aliinase (4,0-6,0 $\mu$ moles ác. pirúvico $\left.\mathrm{g}^{-1}\right)$; para sabor "muito pouco picante" deve apresentar níveis intermediários de atividade da aliinase (4,0-6,0 $\mu$ moles ác. pirúvico $\left.\mathrm{g}^{-1}\right)$ e de acidez (0,3-0,2 g ác. pirúvico $\left.100 \mathrm{~g}^{-1}\right)$, mas alto de sólidos solúveis (>9Brix); e para ser considerada "doce" deve ter alto conteúdo de sólidos solúveis $\left(>9^{\circ}\right.$ Brix $)$, baixa a moderada acidez $\left(<0,2 \mathrm{~g}\right.$ ác. pirúvico $\left.100 \mathrm{~g}^{-1}\right)$ e baixa atividade da aliinase $(<4,0 \mu$ moles ác. pirúvico $\mathrm{g}^{-1}$ ).

Este trabalho teve como objetivo conhecer a evolução da qualidade de cebolas, quando levadas à condição ambiente, depois de armazenadas sob refrigeração.

\section{MATERIAL E MÉTODOS}

Foram utilizados bulbos de cebola 'Superex', tipo comercial (Grupo Amarela, Subgrupo Globular e Classe 2345), produzidos em Monte Alto (SP) na safra de 2003. Estes bulbos foram cultivados em solo que apresentava os parâmetros de fertilidade: $7 \mathrm{~g} \mathrm{dm}^{-3} \mathrm{de}$ matéria orgânica (M.O.); $195 \mathrm{mg} \mathrm{dm}^{-3}$ de P; 3,5 $\mathrm{mmol}_{\mathrm{c}} \mathrm{dm}^{-3}$ de $\mathrm{K} ; 41 \mathrm{mmol}_{\mathrm{c}}$ $\mathrm{dm}^{-3}$ de Ca; 13 mmol $_{\mathrm{c}} \mathrm{dm}^{-3}$ de $\mathrm{Mg} ; 16$ mmol $\mathrm{dm}^{-3}$ de $\mathrm{Al}+\mathrm{H} ; 57,5 \mathrm{mmol} \mathrm{dm}^{-3}$ de soma de bases (SB); 73,5 $\mathrm{mmol}_{\mathrm{c}} \mathrm{dm}^{-3}$ de CTC (capacidade de troca iônica) e saturação de bases (V) igual a 78\%. Os bulbos colhidos em 30/07/03, aos 105 dias após o plantio, foram curados à sombra por cinco dias, destopados, padronizados quanto ao tamanho (diâmetro de 40-55 mm), acondicionados em sacos de rede de fibra plástica $(20 \mathrm{~kg}) \mathrm{e}$ armazenados sob refrigeração $\left(10-12^{\circ} \mathrm{C}\right.$, $68 \%$ UR), por até 91 dias. No início (1 dia) e após 16; 31; 61 e 91 dias, amostras $(20 \mathrm{~kg})$ tomadas ao acaso foram levadas à condição ambiente $\left(22^{\circ} \mathrm{C} \pm 1{ }^{\circ} \mathrm{C}\right.$; $59 \%$ UR). Estes bulbos foram avaliados a cada quatro dias quanto à massa fresca, mediante pesagem em balança Marte mod. AS 2000 e precisão de 0,1 g, e aparência, através da atribuição das notas: 1 = ótima (bulbos túrgidos, sem podridões e com coloração normal); 2 $=$ boa $(5 \%$ dos bulbos com início de descoloração e algum murchamento); 3 = regular $(10-15 \%$ com início de descoloração e murchamento, com até 5\% com início de podridões e brotamentos); 4 = ruim (mais de $50 \%$ com brotações e podridões); 5 = péssima (bulbos totalmente comprometidos).

A qualidade interna dos bulbos foi avaliada a cada sete dias, em amostra de cinco bulbos, através dos parâmetros coloração, teor de sólidos solúveis (SS) e de acidez titulável (AT) e atividade da aliinase.

A coloração do interior dos bulbos foi determinada em cinco bulbos, usando-se um aparelho Minolta Chromameter CR 200b que se expressa pelos valores $\mathrm{L}^{*}, \mathrm{a}^{*}, \mathrm{~b}^{*}$, permitindo caracterizar a coloração através dos parâmetros, luminosidade ( $\mathrm{L}^{*}$ ), ângulo hue ou de cor $\left({ }^{\circ} \mathrm{h}\right)$, determinado através da expressão $\tan ^{-1}\left(\mathrm{~b} * / \mathrm{a}^{*}\right)$ e cromaticidade $(\mathrm{C})$, expressa pela equação $C r=\sqrt{\left(a^{*}\right)^{2}+\left(b^{*}\right)^{2}}$ (Minolta Corp., 1994).

A acidez titulável (AT) foi determinada em quatro repetições, tomando-se $30 \mathrm{~g}$ de material triturado dos bulbos, que foi acrescido de água destilada e titulado com $\mathrm{NaOH}$ a $0,05 \mathrm{~N}$, tendo seu teor expresso em gramas de ácido pirúvico $100 \mathrm{~g}^{-1}$ de polpa (Instituto Adolfo Lutz, 1985).

O conteúdo de sólidos solúveis (SS) foi expresso em ${ }^{\circ}$ Brix, determinado em gotas extraídas da polpa triturada, em quatro repetições, e quantificado em refratômetro digital Atago PR-101, Palette (AOAC, 1997).

$A$ atividade da aliinase foi determinada segundo o proposto por Schwimmer \& Weston (1961), utilizando-se quatro bulbos sem a película superficial. Dois bulbos eram submetidos a aquecimento em microondas, na potência alta por três minutos, antes da determinação (branco). Estes bulbos aquecidos e os outros dois não aquecidos foram triturados e deixados em repouso por $5 \mathrm{~min}$., para que houvesse a sedimentação das partículas de maior peso. De cada material, tomou-se $5 \mathrm{~g}$ do sobrenadante, que foram reagidos com $5 \mathrm{~mL}$ de ácido tricloroacético (TCA) a $0,5 \%$, agitados por uma hora, filtrados em funil de Büchner e tiveram seus volumes completados para $100 \mathrm{~mL}$ com água destilada. Alíquotas destes extratos foram reagidos com 2,4 dinitrofenilhidrazina a $0,0125 \%$ dissolvida em $\mathrm{HCl}$ a $2 \mathrm{M}$, incubadas em banho-maria a $37^{\circ} \mathrm{C}$, por $10 \mathrm{~min}$., e interrompida com $5 \mathrm{~mL}$ de $\mathrm{NaOH}$ a $0,6 \mathrm{M}$, e leitura da absorbância a $420 \mathrm{~nm}$. Utilizou-se como padrão, ácido pirúvico a 2 $\mu \mathrm{mol} \mathrm{mL}^{-1}$ e como branco, água destilada. Os resultados foram calculados pela diferença entre os valores quantificados nos materiais, cru e aquecido, e expressos em micromoles de ácido pirúvico por grama de cebola.

Os bulbos foram classificados quanto à pungência de acordo com o indicado pelo VLI Sweet Index (Vidalialabs, 2004) e quanto ao sabor pelo proposto por Miguel et al. (2004). O VLI Sweet Labs indica que as cebolas podem ser classificadas quanto à pungência através da atividade da aliinase, que é expressa em $\mu$ moles de ácido pirúvicog ${ }^{-1}$, em "muito suave" (0-2,9 $\mu$ moles $\left.\mathrm{g}^{-1}\right)$, "suave" (3,0-4,2 $\mu$ moles $\left.\mathrm{g}^{-1}\right)$, "levemente pungente" (4,3-5,5 $\mu$ moles $\left.g^{-1}\right)$, "pun-

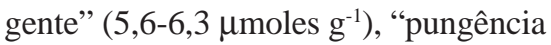
forte" (6,4-6,9 $\mu$ moles $\left.\mathrm{g}^{-1}\right)$, "pungência muito forte" $\left(7,0-7,9 \mu\right.$ moles g $\left.\mathrm{g}^{-1}\right) \mathrm{e}$ "picante" (8,0-10,0 $\mu$ moles $\left.\mathrm{g}^{-1}\right)$. São certificadas como "doces", as cebolas com concentração até "levemente pungente" e como "extra doce", aquelas com concentração até "suave".

O delineamento experimental foi em blocos ao acaso, com 2 a 7 tratamentos (datas de avaliação durante os períodos de armazenamento ao ambiente). A evolução da perda de massa fresca foi analisada através de análise de regressão, pelo teste-t a $1 \%$ de probabilidade (Neter et al., 1978).

\section{RESULTADOS E DISCUSSÃO}

A intensidade da perda de massa fresca durante o período de armazenamento ao ambiente foi influenciada pelo tempo inicial sob refrigeração (Tabela 1), pois até o $31^{\circ}$ dia quanto maior este período, maior a taxa de perda de massa sob a condição ambiente. A perda de massa se constitui em fator limitante ao armazenamento deste produto, pois além de implicar em sérios prejuízos de ordem econômica, é 
indicativo de envelhecimento (Maia et al., 2000). Esta perda deve ser associada à desidratação e à respiração, e o aumento na intensidade foi atribuído ao envelhecimento progressivo dos bulbos, mesmo sob refrigeração. As cebolas mantidas por 91 dias sob refrigeração, apresentavam qualidade ruim quando levadas ao ambiente, que se tornou péssima (nota 5) em quatro dias (Figura 1).

A aparência externa dos bulbos foi muito afetada durante o período ao ambiente (Figura 1). Os bulbos refrigerados por somente um dia ou refrigerados por 16 dias atingiram a aparência regular (nota 3) em 16 e 24 dias, respectivamente, e se conservaram por até 38 dias. Os mantidos sob refrigeração por $31 \mathrm{ou}$ 61 dias mantiveram boa aparência (nota 2) por até 38 dias, enquanto que os estocados por 91 dias foram levados ao ambiente com aparência ruim (nota 4), que chegou a péssima em 4 dias. $\mathrm{O}$ período de até 61 dias de armazenamento refrigerado mostrou-se adequado para estimular a dormência dos bulbos, sem o aparecimento de podridões.

A coloração interna dos bulbos não foi afetada pelo período de armazenamento refrigerado, mas estes, depois de levados à condição ambiente, mostraram tendência não significativa de redução do ângulo de cor e da cromaticidade, ou seja, de coloração mais amarelada (Tabela 2). Apesar da ocorrência das diferenças entre os dias de avaliação, o que pode ser atribuído às características individuais, os valores apresentados reafirmam a manutenção da boa qualidade visual da polpa (Kader, 2002). Este amarelecimento também foi observado em cebolas 'Granex-33-Híbrida', armazenadas à temperatura ambiente, com diminuição na luminosidade ou leve escurecimento em cebolas 'Baia Periforme' (Yokomizo et al., 1977) o que não aconteceu neste experimento.

Os teores de sólidos solúveis e de acidez titulável dos bulbos não foram afetados pelo armazenamento refrigerado mas, quando levados ao ambiente, a tendência foi de redução nos SS e de incremento na AT, como resultado de aumento na temperatura de armazenamento (Tabela 2), o que também foi indicado por Chitarra \& Chitarra

Tabela 2. Coloração e características químicas de bulbos de cebola 'Superex" mantidos sob refrigeração $\left(10-12^{\circ} \mathrm{C}, 68 \% \mathrm{UR}\right)$, quando levados ao ambiente $\left(22^{\circ} \mathrm{C}, 59 \% \mathrm{UR}\right)$ (Color and chemical characteristics of onion bulbs, cv. 'Superex' stored under refrigeration $\left(10-12^{\circ} \mathrm{C}, 68 \%\right.$ UR) and stored under environmental conditions $\left(22^{\circ} \mathrm{C}, 59 \%\right.$ UR)). Jaboticabal, UNESP, 2004.

\begin{tabular}{|c|c|c|c|c|c|c|}
\hline \multirow{2}{*}{$\begin{array}{l}\text { Ambiente } \\
\text { (dia) }\end{array}$} & \multicolumn{3}{|c|}{ Coloração } & \multirow{2}{*}{$\begin{array}{l}\text { Sólidos } \\
\text { solúveis } \\
\text { ( }{ }^{\circ} \text { Brix) }\end{array}$} & \multirow{2}{*}{$\begin{array}{l}\text { Acidez titulável } \\
\text { (g ác. pirúvico } \\
100 \mathrm{~g}^{-1} \text { ) }\end{array}$} & \multirow{2}{*}{$\begin{array}{l}\text { Pungência } \\
\text { (momoles de ác. } \\
\text { pirúvico } \text { g }^{-1} \text { ) }\end{array}$} \\
\hline & $\mathrm{L}^{*}$ & $h^{\circ}$ & C & & & \\
\hline \multicolumn{7}{|c|}{ Refrigeração (1 dia) } \\
\hline$\overline{0}$ & $72,4 \mathrm{a}$ & $120,0 \mathrm{a}$ & $4,7 \mathrm{a}$ & $8,4 a b$ & $0,152 b$ & 5,816 a \\
\hline 7 & $72,7 \mathrm{a}$ & 115,5 a & $5,2 \mathrm{a}$ & $8,6 a b$ & $0,134 \mathrm{c}$ & $7,115 a$ \\
\hline 15 & $71,7 \mathrm{a}$ & 113,4 a & $5,0 \mathrm{a}$ & 9,0 a & $0,132 \mathrm{c}$ & 5,938 a \\
\hline 21 & $72,6 \mathrm{a}$ & 112,9 a & 4,4 a & $8,2 a b$ & $0,152 \mathrm{~b}$ & 6,069 a \\
\hline 27 & $72,3 \mathrm{a}$ & $110,7 \mathrm{a}$ & $3,5 \mathrm{a}$ & $7,8 \mathrm{~b}$ & $0,169 a$ & 6,907 a \\
\hline $\mathrm{CV}(\%)$ & 3,85 & 5,87 & 32,38 & 6,74 & 3,45 & 10,10 \\
\hline \multicolumn{7}{|c|}{ Refrigeração (16 dias) } \\
\hline 0 & $73,6 a b$ & $122,0 \mathrm{a}$ & $4,5 \mathrm{a}$ & $8,2 a b$ & $0,154 \mathrm{bc}$ & $6,748 a b$ \\
\hline 7 & $70,4 \mathrm{~b}$ & $121,5 \mathrm{a}$ & $4,6 \mathrm{a}$ & $7,6 \mathrm{bc}$ & $0,160 \mathrm{a}$ & $4,656 \mathrm{~b}$ \\
\hline 15 & $70,7 \mathrm{ab}$ & 116,7 a & $3,2 \mathrm{a}$ & $7,6 \mathrm{c}$ & $0,160 a b$ & $5,951 \mathrm{ab}$ \\
\hline 21 & $73,5 a b$ & $120,4 \mathrm{a}$ & $4,0 \mathrm{a}$ & $8,2 \mathrm{abc}$ & $0,152 \mathrm{c}$ & $6,784 a b$ \\
\hline 27 & $74,0 \mathrm{ab}$ & $119,0 \mathrm{a}$ & $3,8 \mathrm{a}$ & $7,8 \mathrm{bc}$ & $0,160 a b$ & $7,108 \mathrm{a}$ \\
\hline 33 & 75,3 a & 121,9 a & $3,7 \mathrm{a}$ & $7,6 \mathrm{c}$ & $0,160 \mathrm{a}$ & 7,117 a \\
\hline 39 & $75,2 a b$ & $120,6 \mathrm{a}$ & $4,2 \mathrm{a}$ & $8,2 \mathrm{a}$ & $0,150 \mathrm{c}$ & $7,225 \mathrm{a}$ \\
\hline CV (\%) & 2,34 & 2,75 & 14,63 & 2,79 & 1,41 & 8,27 \\
\hline \multicolumn{7}{|c|}{ Refrigeração (31 dias) } \\
\hline 0 & $76,5 \mathrm{a}$ & $128,9 \mathrm{a}$ & $4,2 \mathrm{a}$ & $9,0 \mathrm{a}$ & $0,135 \mathrm{~b}$ & $6,209 a$ \\
\hline 7 & $74,2 \mathrm{a}$ & 122,5 a & $4,0 \mathrm{a}$ & $7,7 \mathrm{~b}$ & 0,159 a & 7,370 a \\
\hline 15 & $75,9 \mathrm{a}$ & $121,9 \mathrm{a}$ & $4,4 \mathrm{a}$ & $7,8 \mathrm{c}$ & $0,138 b$ & $8,285 \mathrm{a}$ \\
\hline 21 & $72,8 \mathrm{a}$ & $119,1 \mathrm{a}$ & $4,3 \mathrm{a}$ & $7,8 \mathrm{c}$ & 0,163 a & $6,471 \mathrm{a}$ \\
\hline 27 & $75,8 \mathrm{a}$ & $118,3 \mathrm{a}$ & $4,2 \mathrm{a}$ & $7,7 \mathrm{c}$ & 0,164 a & 6,376 a \\
\hline 33 & $75,1 \mathrm{a}$ & $117,5 \mathrm{a}$ & $4,0 \mathrm{a}$ & $7,9 \mathrm{bc}$ & $0,159 a$ & 5,666 a \\
\hline 39 & $73,0 \mathrm{a}$ & $119,0 \mathrm{a}$ & $3,7 \mathrm{a}$ & $7,8 \mathrm{c}$ & $0,161 \mathrm{a}$ & 8,636 a \\
\hline $\mathrm{CV}(\%)$ & 3,95 & 3,72 & 15,20 & 1,63 & 1,66 & 12,28 \\
\hline \multicolumn{7}{|c|}{ Refrigeração (61 dias) } \\
\hline 0 & $75,4 \mathrm{a}$ & $122,5 \mathrm{a}$ & $4,8 \mathrm{a}$ & $8,5 \mathrm{a}$ & $0,152 \mathrm{~b}$ & $8,204 a$ \\
\hline 7 & $76,0 \mathrm{a}$ & 119,8 a & $4,5 \mathrm{a}$ & $7,9 a b$ & $0,156 b$ & 8,486 a \\
\hline 15 & 75,9 a & $118,1 \mathrm{a}$ & $3,0 \mathrm{~b}$ & $7,9 a b$ & $0,158 a b$ & 8,133 a \\
\hline 21 & $74,0 \mathrm{a}$ & $115,4 a$ & $3,2 \mathrm{~b}$ & $7,6 \mathrm{bc}$ & $0,138 \mathrm{bc}$ & $6,383 b$ \\
\hline 27 & $71,4 \mathrm{a}$ & 115,9 a & $3,5 \mathrm{~b}$ & $7,2 \mathrm{c}$ & $0,165 \mathrm{c}$ & $8,001 \mathrm{a}$ \\
\hline 33 & $71,0 \mathrm{a}$ & $116,0 \mathrm{a}$ & $3,3 \mathrm{~b}$ & $7,5 \mathrm{bc}$ & $0,172 b c$ & 8,467 a \\
\hline 39 & $70,8 \mathrm{a}$ & $114,0 \mathrm{a}$ & $3,5 \mathrm{~b}$ & $7,8 a b$ & $0,163 a b$ & $7,199 a b$ \\
\hline $\mathrm{CV}(\%)$ & 3,91 & 3,79 & 18,79 & 3,17 & 3,65 & 7,01 \\
\hline \multicolumn{7}{|c|}{ Refrigeração (91 dias) } \\
\hline 0 & $79,0 \mathrm{a}$ & $112,8 \mathrm{a}$ & $4,9 a$ & $7,0 \mathrm{~b}$ & $0,165 \mathrm{a}$ & $10,989 a$ \\
\hline 7 & $72,1 \mathrm{~b}$ & $113,2 \mathrm{a}$ & $5,1 \mathrm{a}$ & $7,6 \mathrm{a}$ & $0,146 \mathrm{~b}$ & $8,033 \mathrm{a}$ \\
\hline CV (\%) & 3,76 & 3,52 & 9,80 & 4,47 & 3,89 & 6,89 \\
\hline
\end{tabular}

$\mathrm{L}^{*}=$ luminosidade $; \mathrm{h}^{\circ}=$ ângulo de cor; $\mathrm{C}=$ cromaticidade; Pungência = atividade da aliinase $\left(\mu\right.$ moles de ác. pirúvico $\left.\mathrm{g}^{-1}\right) ; \mathrm{CV}=$ coeficiente de variação $\left(\mathrm{L}^{*}=\right.$ luminosity; $\mathrm{h}^{\circ}=$ color angle; $\mathrm{C}=$ cromaticity; Pungency $=$ aliinase activity (mmoles of piruvic acid $\mathrm{g}^{-1}$ ); $\mathrm{CV}=$ variation coeficient).

(1990). Fontes (1998) também observou que o teor de sólidos solúveis de cebolas pode ser afetado pelo período e condições do armazenamento e pela cultivar, enquanto Currah \& Proctor (1990) relataram aumento na acidez com o avanço do período de armazenamento.

A pungência aumentou durante o período de armazenamento refrigerado inicial, de 5,816 mmoles $\mathrm{g}^{-1}$ para 10,989 mmoles $\mathrm{g}^{-1}$ após 91 dias (Tabela 2), ou seja, alterou o sabor das cebolas de "pun- 
gente" para "picante" (Vidalialabs, 2004), indicando o efeito protetor da aliinase na conservação dos bulbos, uma vez que sempre se analisou aqueles adequados ao consumo. Quando levados para condições de ambiente, a atividade da aliinase não mudou significativamente.

Wall \& Corgan (1992) relatam que a relação entre os teores de SS, AT e pungência pode ser uma melhor indicação da qualidade organoléptica dos bulbos, que a análise isolada destes parâmetros. Tendo-se esta condição, as cebolas durante o período refrigerado ou depois de levadas ao ambiente puderam ser classificadas como "picantes", devido à alta atividade da aliinase $(>6,0$ $\mu$ moles ác. pirúvico $\mathrm{g}^{-1}$ ), e teores de AT e de SS intermediários $(<0,2 \mathrm{~g}$ ác. pirúvico $100 \mathrm{~g}^{-1} \mathrm{e} » 8^{\circ}$ Brix) (Miguel et al., 2004).

O armazenamento de cebola 'Superex' a $10-12^{\circ} \mathrm{C}$ e $68 \%$ UR permite sua conservação por até 61 dias, sem que os bulbos apresentem variações significativas nas suas características originais, mas com aumento na pungência. Estes bulbos, quando levados ao ambiente $\left(22^{\circ} \mathrm{C}, 59 \%\right.$ UR) mantiveram qualidade adequada por até 38 dias.

\section{AGRADECIMENTOS}

Os autores agradecem à FAPESP pela bolsa de iniciação científica concedida ao primeiro autor.

\section{LITERATURA CITADA}

ANDRADE MO; LIMA UA. 1983. Cebola - produção, pré-processamento e transformação agroindustrial. In: MARTINELLI FILHO, A. et al. (eds). Processamento e tecnologia dos alimentos. Piracicaba: ESALQ/USP, p. 73-115.

AOAC. 1997. Official methods of analysis of the Association of Official Analytical Chemists International. Washington: AOAC. p. 16-45.

CHITARRA MIF; CHITARRA AB. 1990. Póscolheita de frutos e hortaliças: fisiologia $e$ manuseio. Lavras: ESAL/FAEPE. 320p.

CURRAH L; PROCTOR F. 1990. Onions in tropical regions. Natural Resources Institute 35: 144-163.

FONTES PCR. 1998. Cultura da cebola. Viçosa: UFV. 40p.

INSTITUTO ADOLFO LUTZ. 1985. Normas analíticas: métodos químicos e físicos para análises de alimentos. São Paulo: IAL. 371p.

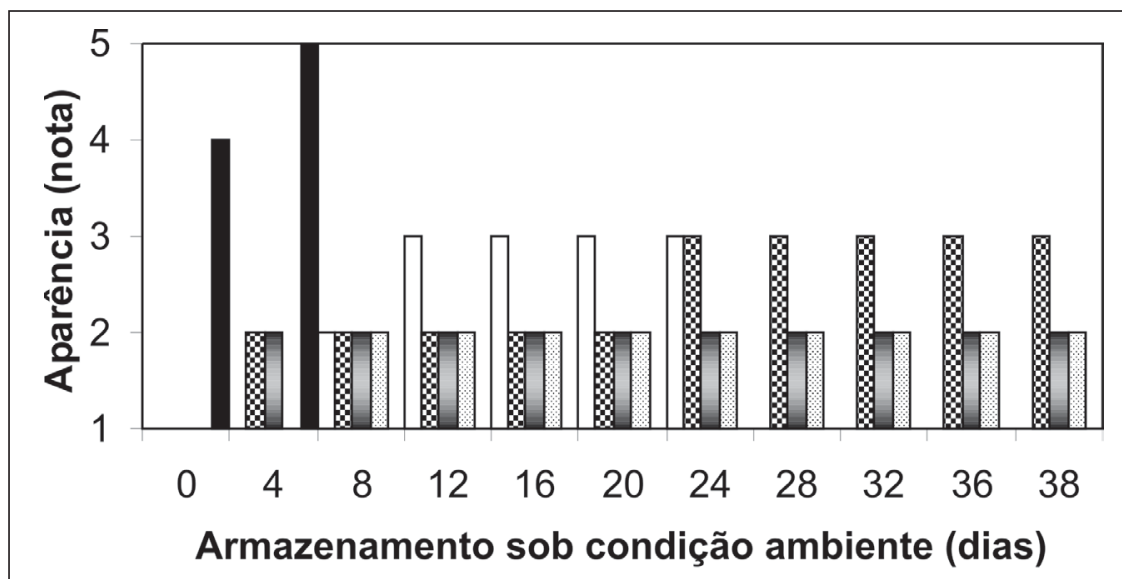

\section{Dia $\approx 16$ Dias $\square 31$ Dias $\square 61$ Dias $\square 91$ Dias}

Figura 1. Evolução da aparência em bulbos de cebola 'Superex' mantidos sob condição de refrigeração $\left(10-12^{\circ} \mathrm{C}, 68 \%\right.$ UR) quando levados à condição de ambiente $\left(22^{\circ} \mathrm{C}, 59 \% \mathrm{UR}\right)$. (Notas: 1 = ótima; 5 = péssima) (Evolution in the appearance of onion bulbs, cv. 'Superex', stored under refrigeration $\left(10-12^{\circ} \mathrm{C}, 68 \% \mathrm{UR}\right)$ and stored under environmental conditions $\left(22^{\circ} \mathrm{C}, 59 \%\right.$ UR). (Grades: 1 = very good; 5 = very bad)). Jaboticabal, UNESP, 2004.

Tabela 1. Evolução da perda de massa fresca pelos bulbos de cebola 'Superex' mantidos sob refrigeração $\left(10-12^{\circ} \mathrm{C}, 68 \% \mathrm{UR}\right)$, quando levados à condição de ambiente $\left(22^{\circ} \mathrm{C}, 59 \%\right.$ UR) (Evolution of fresh mass lost of onion bulbs, cv. 'Superex', stored under refrigeration $\left(10-12^{\circ} \mathrm{C}, 68 \% \mathrm{UR}\right)$, and transferred to environmental conditions $\left.\left(22^{\circ} \mathrm{C}, 59 \% \mathrm{UR}\right)\right)$. Jaboticabal, UNESP, 2004.

\begin{tabular}{cc}
\hline Tempo de refrigeração (dias) & Equações de perda de massa fresca \\
\hline 1 & $Y=0,7171+0,1331^{* *} X ; R=0,9705$ \\
16 & $Y=0,2234+0,1463^{* *} X ; R=0,9984$ \\
31 & $Y=0,0166+0,1992^{* *} X ; R=0,9940$ \\
61 & $Y=-0,2049+0,1999^{* *} X ; R=0,994$ \\
91 & - \\
\hline
\end{tabular}

$\mathrm{Y}=\%$ da massa inicial; $\mathrm{X}=$ dias de armazenamento. $* *$ t significativo a $1 \%$ de probabilidade ( $\mathrm{Y}=\%$ of initial mass; $\mathrm{X}=$ days of storage. $* *$ t significative at $1 \%$ probability).

KADER AA. 2002. Postharvest technology of horticultural crops. Davis: University of California. 535p.

KOPSELL DE; RANDLE WM. 1997. Onion cultivars in differ pungency and bulb quality changes during storage. HortScience 32: 12601263.

MAIA MCC; PEDROSA JF; TORRES FILHO J; NEGREIROS MZ de; BEZERRA NETO F. 2000. Características de qualidade de cebola múltipla durante armazenamento sob condição ambiental não controlada. Horticultura Brasileira 18: 61-64.

MIGUEL ACA; DURIGAN MFB; DURIGAN JF; MORETTI CL. 2004. Postharvest quality of twelve onion cultivars grown in the southeast of Brazil. In: INTERNATIONAL ISHS SYMPOSIUM ON EDIBLE ALLIACEAE, 4. Abstracts... Beijing: ISEA. p. 235.

MINOLTA CORP. 1994. Precise color communication: color control from feeling to instrumentation. Ramsey: Minolta Corporation Instrument Systems Division. 49p.
NETER J; WASSERMAN W; WHITMORE GA. 1978. Applied linear statistical models. Massachussetts: Alynand and Bacon. 745p.

OLIVEIRA VR. Cebola doce. Horticultura Brasileira 22: s.n. (Reportagem da contra capa).

RANDLE WM; BUSSARD ML. 1993. Pungency and sugars of short-day onions as affected by sulfur nutrition. Journal of the American Society for Horticultural Science 118: 766770.

RANDLE WM; BLOCK E; LITTLEJOHN MH; PUTMAN D; BUSSARD ML. 1994. Onion (Allium cepa L.) thiosulfinates respond to increasing sulfur fertility. Journal of Agriculture and Food Chemistry 42: 20852088.

RUBATZKY VE; YAMAGUCHI M. 1997. World vegetables. Principles, production and $843 \mathrm{p}$.

SCHWIMMER S; WESTON WJ. 1961. Enzymatic development of pyruvic acid in onion as a measure of pungency. Journal of Agricultural and Food Chemistry 9: 301-304. nutritive values. New York: Chapman \& Hall. 
VARELA et al. 1980. Cebola: auto-suficiência e normalização do abastecimento. Informe Agropecuário 62: 65-70.

VIDALIALABS - VLI Sweet index (Sweetometer). 2004, 7 de outubro. Disponível em http://www.vidalialabs.com/images/ sweeetometer.jpg/

VILELA NJ; MAKISHIMA N; OLIVEIRA VR; COSTA ND; MADAIL JCM; CAMARGO FILHO W; BOEING G; MELO PCT. 2005. Desafios e oportunidades para o agronegócio da cebola no Brasil. Horticultura Brasileira 23: 1029-1033.
WALL MM; CORGAN JN. 1992. Relationship between pyruvate analysis and flavor perception for pungency determination. HortScience 27: 1029-1030.
YOKOMIZO Y; DRAETTA IS; COSTA RB; GARCIA JL. 1977. Composição química de diferentes variedades de cebola (Allium cepa L.) e sua caracterização. Coletânea do Instituto de Tecnologia de Alimentos 8: 501-517. 